\title{
Innovative Biomaterials Based on Collagen-Hydroxyapatite and Doxycycline for Bone Regeneration
}

\author{
Narcisa Mederle, ${ }^{1}$ Stefania Marin, ${ }^{2}$ Minodora Maria Marin, ${ }^{2}$ Elena Danila, \\ Ovidiu Mederle, ${ }^{3}$ Madalina Georgiana Albu Kaya, ${ }^{2}$ and Mihaela Violeta Ghica ${ }^{4}$ \\ ${ }^{1}$ Faculty of Veterinary Medicine, Banat's University of Agricultural Sciences, 119 Calea Aradului, 300645 Timişoara, Romania \\ ${ }^{2}$ Collagen Department, Leather and Footwear Research Institute, 93 Ion Minulescu Street, 031215 Bucharest, Romania \\ ${ }^{3}$ Department of Histology, Faculty of Medicine, University of Medicine and Pharmacy "Victor Babes", Piata Eftimie Murgu 2, \\ 300041 Timişoara, Romania \\ ${ }^{4}$ Department of Physical and Colloidal Chemistry, Faculty of Pharmacy, "Carol Davila” University of Medicine and Pharmacy, \\ 6 Traian Vuia Street, 020956 Bucharest, Romania
}

Correspondence should be addressed to Madalina Georgiana Albu Kaya; albu_mada@yahoo.com

Received 4 February 2016; Accepted 3 March 2016

Academic Editor: Mikhael Bechelany

Copyright (C) 2016 Narcisa Mederle et al. This is an open access article distributed under the Creative Commons Attribution License, which permits unrestricted use, distribution, and reproduction in any medium, provided the original work is properly cited.

Bone regeneration is a serious challenge in orthopedic applications because of bone infections increase, tumor developing, and bone loss due to trauma. In this context, the aim of our study was to develop innovative biomaterials based on collagen and hydroxyapatite $(25,50$, and $75 \%)$ which mimic bone composition and prevent or treat infections due to doxycycline content. The biomaterials were obtained by freeze-drying in spongious forms and were characterized by water uptake capacity and microscopy. The in vitro release of doxycycline was also determined and established by non-Fickian drug transport mechanism. Among the studied biomaterials, the most suitable one to easily deliver the drug and mimic bone structure, having compact structure and lower capacity to uptake water, was the one with $75 \%$ hydroxyapatite and being cross-linked.

\section{Introduction}

Bone tissue is a multilayered composite which contains an inorganic phase embedded in a collagen based organic matrix (type I collagen and proteoglycans) [1,2]. The inorganic phase is composed mostly of calcium phosphates, the main component being hydroxyapatite $\left(\mathrm{Ca}_{10}\left(\mathrm{PO}_{4}\right)_{6}(\mathrm{OH})_{2}\right)$ [3]. The biological hydroxyapatites which are found in hard tissues are rod-like or plate-like crystals of variable lengths $(30-45 \mathrm{~nm})$ oriented parallel to the collagen fibrils in which different cell types (osteoblasts, osteoclasts, and osteocytes) reside [4].

Due to bone infections increase, tumor developing, and bone loss due to trauma, bone regeneration is a serious challenge in orthopedic applications. Current therapies include autografts, allografts, and other bone substitutes [5]. Autografts (bone tissue obtained from another anatomical site in the same subject) are the best treatment for bone defects but the procedure involves risks of donor site like morbidity, structural and anatomical complications, and graft sorption [6]. When autografts cannot be used, allografts (tissue obtained from members of the same species) are considered but this comes with its own disadvantages, immunological response and possible disease transmission [7].

Bone tissue engineering can offer an alternative by providing biointegrating scaffolds in which living cells can develop. The scaffolds for bone tissue engineering should possess several properties such as biocompatibility, mechanical similarities with the target tissue and high porosity for cell adhesion, proliferation, and tissue development [8-10]. The ideal scaffold mimics the structural and biological functions of a naturally occurring extracellular matrix in terms of physical structure and chemical composition [11,12].

Among natural polymers, collagen is the most abundant protein in mammals, the main source being bovine tissue $[13,14]$. Collagen scaffolds used in tissue engineering are in the form of sponges, thin sheets (membrane), or gel/hydrogel 
TABLE 1: Composition and name of collagen gels.

\begin{tabular}{lcccc}
\hline Code of gels & Collagen, $\%$ & Hydroxyapatite, $\%$ & Doxycycline, $\%$ & Glutaraldehyde, $\%$ \\
\hline CHD1 & 1 & 25 & 0.2 & 0 \\
CHDR1 & 1 & 25 & 0.2 & 0.25 \\
CHD2 & 1 & 50 & 0.2 & 0 \\
CHDR2 & 1 & 50 & 0.2 & 0.25 \\
CHD3 & 1 & 75 & 0.2 & 0 \\
CHDR3 & 1 & 75 & 0.2 & 0.25 \\
\hline
\end{tabular}

matrices [15]. Collagen is suited for tissue regeneration due to proper pore structure, permeability, hydrophilicity, and in vivo stability [16].

Hydroxyapatite is a naturally occurring substance that is found in teeth, bones, and tendons, being responsible for the mechanical properties of these tissues. The greatest advantage of hydroxyapatite as a biomaterial is that the host tissue will form direct chemical bonds without the formation of an inert interface which in turn leads to faster and better tissue integration [17].

Doxycycline is a broad spectrum antibiotic from the tetracycline antibiotics group. It is used in the treatment of both Gram-positive and Gram-negative bacteria and inhibits the bacterial protein biosynthesis $[18,19]$. Doxycycline application promotes bone growth in periodontal disease and periimplantitis [20]. Recently, better tissue regeneration has been achieved in the management of induced femur trauma by combining doxycycline and collagen membranes [21].

Regenerative medicine has recently focused on developing biomaterials with multiple functions in order to solve at the same time bone tissue regeneration and infection treatment of prevention. Our research group developed some biomaterials based only on collagen and doxycycline for cell cultures $[22,23]$, tested on animals [21], human clinical trials [24], and also some scaffolds with biovitroceramics (article in press). In this study we developed and characterized some collagen-doxycycline with different amount of hydroxyapatite biomaterials in order to be used in bone defects.

\section{Materials and Methods}

The type I fibrillar collagen gel having a concentration of $2.46 \%(w / w)$ was extracted from calf hide by the technology currently used in our laboratory [16]. Doxycycline hyclate was purchased from Sigma-Aldrich, China, and hydroxyapatite from Sigma-Aldrich, Germany. Sodium hydroxide and phosphate buffer solution, $\mathrm{PBS}(\mathrm{pH}=7.4)$, were of analytical grade.

A value of $0.2 \%$ doxycycline hyclate (reported as being collagen of gel form) was embedded under mechanical stirring into collagen gel having an initial $\mathrm{pH}$ of 2.5. The hydroxyapatite in proportions of 25,50 , and $75 \%$ was added to collagen-doxycycline gel to obtain gels with $1 \%$ collagen and $\mathrm{pH}$ of 7.4. Then some samples were cross-linked with $0.25 \%$ glutaraldehyde (reported as being collagen of dry substance) and some samples were not cross-linked as Table 1 presented.

The collagen gels were freeze-dried using Delta 2-24 LSC (Martin Christ, Germany) lyophilizer and spongious forms were obtained and characterized by microscopy, water uptake, and in vitro release of doxycycline.

2.1. Optical Microscopy Study. All images were captured with a Leica Stereomicroscope model S8AP0, 20-160x magnification capacity. For better evaluation of samples, a $20 \mathrm{x}$ magnification and incident external cold light were used.

2.2. Water Absorption. With a design to determine the water absorption, the samples were first immersed in water at $36^{\circ} \mathrm{C}$. At certain time ranges, the samples were removed from the water and weighed. The water absorption was calculated using the following equation:

$$
\% \text { Water up-take }=\frac{(W t-W d)}{W d}(\mathrm{~g} / \mathrm{g}),
$$

where $W t$ is the weight of the wet samples at immersion time $t$ and $W d$ is the weight of dry samples. All the samples were analyzed in triplicate.

2.3. Drug Release Kinetics Study. The doxycycline hyclate released from the designed composites was investigated using a transdermal sandwich device adapted to a dissolution apparatus fitted with paddle stirrers (Essa Dissolver, Italy) as detailed in our previous studies $[22,25]$. The Peppas law model was applied for the assessment of drug release kinetics:

$$
\frac{m_{t}}{m_{\infty}}=k \cdot t^{n},
$$

where $m_{t} / m_{\infty}$ is the fraction of drug released at time $t, k$ is the kinetic constant, and $n$ is the release exponent in relation to the drug transport mechanism.

\section{Results and Discussion}

Porous 3D collagen based sponges, obtained after lyophilization and having collagen, hydroxyapatite, and doxycycline in composition, cross-linked and non-cross-linked, were analyzed in comparison with the results obtained in our previous work [25].

The surfaces of cross-linked samples are showed in Figure 1. Optical microscopy revealed that the structure is characterized by interconnected pores. It can be seen that pore size decreases for samples with the hydroxyapatite amount increase. Also, the sample CHDR2 presents a more pronounced honey-comb-like structure than the others. 


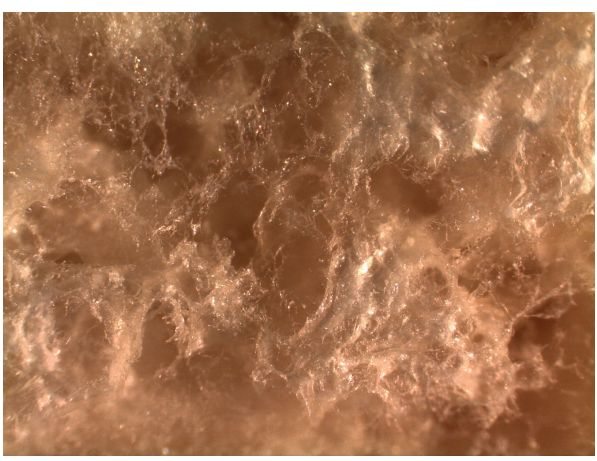

(a)

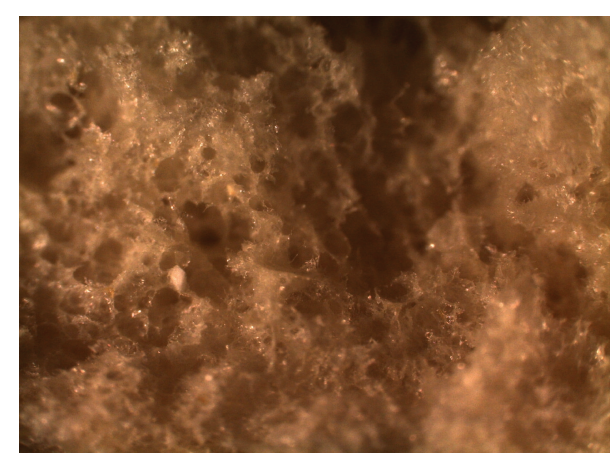

(b)

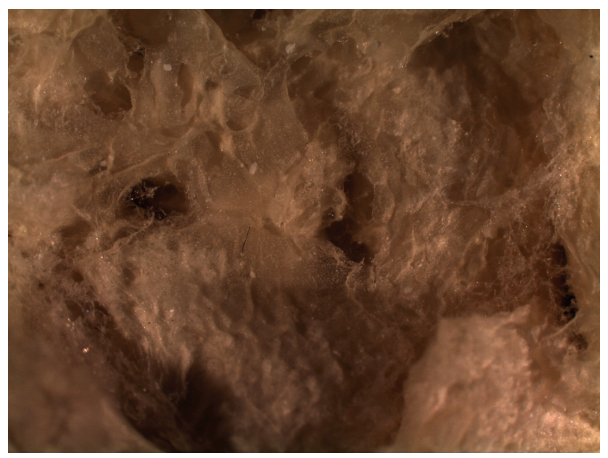

(c)

FIGURE 1: Optical microscopy for cross-linked samples with 25\% (a), 50\% (b), and 75\% (c) hydroxylapatite.

The most compact sponge is the one with the highest amount of hydroxyapatite.

All the matrices showed structures with favorable characteristics for osteointegration. Due to the presence of hydroxyapatite amount and due to pore distribution, osseous cell integration is allowed. Therefore, osseous healing may be accelerated by the presence of hydroxyapatite.

The water uptake for all the studied samples is presented in Figure 2. The changes in sample structures after the water absorption can also be seen.

The water uptake process was analyzed for 72 hours for each sample. It can be noticed that samples without hydroxyapatite absorbed a higher amount of water. Due to the porous structure formed during cross-linking, the samples with glutaraldehyde absorbed a higher amount of water than the non-cross-linked ones. Also, we can see the influence of hydroxyapatite, meaning that the CHDR2 absorbed a bigger quantity of water. So, the most absorbent sponge is the reference one; then, the water uptake capacity decreases with the increase of hydroxyapatite amount. The results obtained from optical microscopy are in correlation with the results obtained in case of water uptake analysis, suggesting that the most compact sample obtained is the one with $75 \%$ hydroxyapatite.

The doxycycline release was investigated and the kinetic patterns were represented as cumulative drug percent released versus time (Figure 3 ).

As it can be seen from Figure 3 both cross-linking agent and amount of HA influence the drug percent released. For all

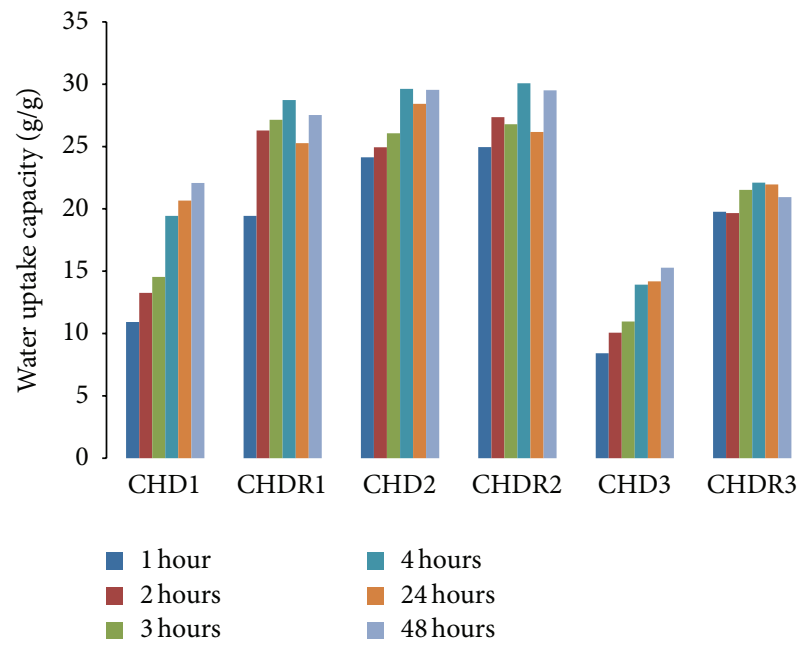

FIGURE 2: The water uptake for biomaterials.

spongious forms, cross-linking determined a decrease of $\mathrm{DH}$ released percent of about 1.08-1.31 times.

The drug released percent was higher for samples with 75\% HA, both non-cross-linked and cross-linked with GA. For non-cross-linked samples the smallest released percent was recorded for the one with $0.50 \%$ HA (about 1.27 times), while for cross-linked samples the smallest, released percent was obtained for the one with $0.25 \%$ HA (about 1.42 times). For both non-cross-linked and cross-linked spongious forms, 
TABle 2: The kinetic parameters and the correlation coefficient specific to Peppas model.

\begin{tabular}{lccc}
\hline Collagen-doxycycline sponges & Kinetic constant $\left(1 / \mathrm{min}^{n}\right)$ & Release exponent & Correlation coefficient \\
\hline CHD1 & 0.015 & 0.593 & 0.9974 \\
CHDR1 & 0.005 & 0.704 & 0.9983 \\
CHD2 & 0.011 & 0.626 & 0.9991 \\
CHDR2 & 0.009 & 0.643 & 0.9982 \\
CHD3 & 0.018 & 0.591 & 0.9979 \\
CHDR3 & 0.012 & 0.647 & 0.9897 \\
\hline
\end{tabular}

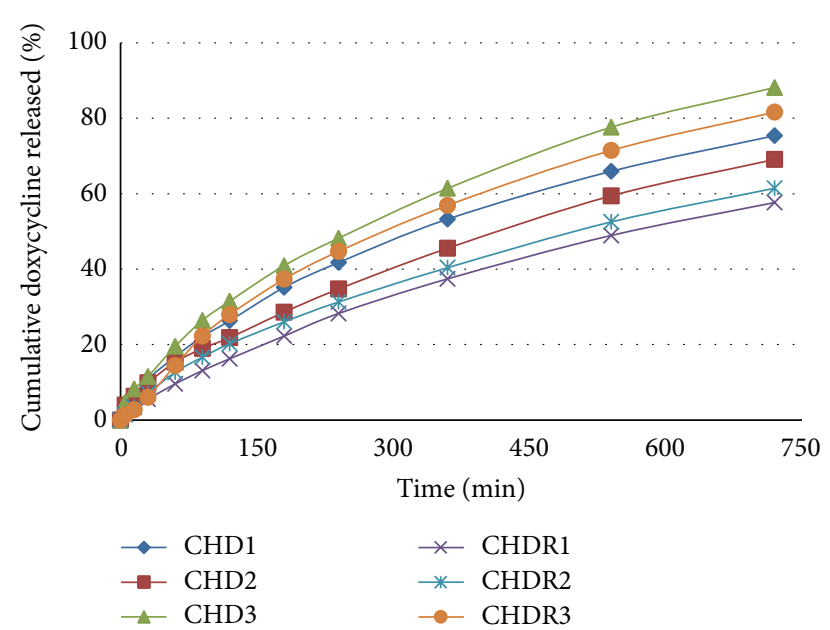

Figure 3: Cumulative release profiles of doxycycline hyclate from biomaterials over time.

the addition of $75 \%$ HA determined a marked increase of DH percent release in comparison with samples with $25 \%$ HA or $50 \% \mathrm{HA}$, respectively.

It can be noticed that the burst release effect is about maximum $30 \%$ in the first two hours of the experiment.

The Peppas model best fitted the experimental kinetic data, suggesting an anomalous non-Fickian drug transport mechanism. The values specific to this model are listed in Table 2.

\section{Conclusions}

3D porous scaffolds based on collagen, doxycycline, and different amount of hydroxyapatite, cross-linked and noncross-linked, were prepared by freeze-drying method. The morphology of the biomaterials determined by water uptake and optical microscopy showed that a higher amount of hydroxyapatite makes more compact structures with less ability of water uptake. The doxycycline release was investigated and the Peppas model best fitted the experimental kinetic data, suggesting an anomalous non-Fickian drug transport mechanism. The combination between bone components (collagen and hydroxyapatite) and antimicrobials (doxycycline) recommends this new innovative product as a promising biomaterial for bone tissue engineering.

\section{Competing Interests}

The authors declare that they have no competing interests.

\section{Acknowledgments}

The authors would like to acknowledge the financial support from the UEFISCDI through PN II 201/2014 (ZettaSkin acronym).

\section{References}

[1] W. S. S. Jee, "Integrated bone tissue physiology: anatomy and physiology," in Bone Mechanics Handbook, S. C. Cowin, Ed., CRC Press, Boca Raton, Fla, USA, 2001.

[2] G. S. Baht, G. K. Hunter, and H. A. Goldberg, "Bone sialoprotein-collagen interaction promotes hydroxyapatite nucleation," Matrix Biology, vol. 27, no. 7, pp. 600-608, 2008.

[3] A. Singh, "Hydroxyapatite, a biomaterial: its chemical synthesis, characterization and study of biocompatibility prepared from shell of garden snail, Helix aspersa," Bulletin of Materials Science, vol. 35, no. 6, pp. 1031-1038, 2012.

[4] R. Z. LeGeros, "Calcium phosphate-based osteoinductive materials," Chemical Reviews, vol. 108, no. 11, pp. 4742-4753, 2008.

[5] D. Puppi, E. Chiellini, F. Chiellini, and A. M. Piras, "Polymeric materials for bone and cartilage repair," Progress in Polymer Science, vol. 35, no. 4, pp. 403-440, 2010.

[6] N. K. Nga, T. T. Hoai, and P. H. Viet, "Biomimetic scaffolds based on hydroxyapatite nanorod/poly $(\mathrm{D}, \mathrm{L})$ lactic acid with their corresponding apatite-forming capability and biocompatibility for bone-tissue engineering," Colloids and Surfaces B: Biointerfaces, vol. 128, pp. 506-514, 2015.

[7] C. R. Perry, "Bone repair techniques, bone graft, and bone graft substitutes," Clinical Orthopaedics and Related Research, no. 360, pp. 71-86, 1999.

[8] K. Alvarez and H. Nakajima, "Metallic scaffolds for bone regeneration," Materials, vol. 2, no. 3, pp. 790-832, 2009.

[9] M. Ficai, E. Andronescu, G. Voicu, D. Ficai, M. G. Albu, and A. Ficai, "Mollusc shell/collagen composite as potential biomaterial for bone substitutes," Romanian Journal of Materials, vol. 40, no. 4, pp. 359-364, 2010.

[10] I. O. Smith, X. H. Liu, L. A. Smith, and P. X. Ma, "Nanostructured polymer scaffolds for tissue engineering and regenerative medicine," Wiley Interdisciplinary Reviews: Nanomedicine and Nanobiotechnology, vol. 1, no. 2, pp. 226-236, 2009.

[11] D. W. Hutmacher, "Scaffolds in tissue engineering bone and cartilage," Biomaterials, vol. 21, no. 24, pp. 2529-2543, 2000. 
[12] R. Zhang and P. X. Ma, "Synthetic nano-fibrillar extracellular matrices with predesigned macroporous architectures," Journal of Biomedical Materials Research, vol. 52, no. 2, pp. 430-438, 2000.

[13] K. Gelse, E. Pöschl, and T. Aigner, "Collagens-structure, function, and biosynthesis," Advanced Drug Delivery Reviews, vol. 55, no. 12, pp. 1531-1546, 2003.

[14] M. G. Patino, M. E. Neiders, S. Andreana, B. Noble, and R. E. Cohen, "Collagen: an overview," Implant Dentistry, vol. 11, no. 3, pp. 280-285, 2002.

[15] M. G. Albu, I. Titorencu, and M. V. Ghica, "Collagen-based drug delivery systems for tissue engineering," in Biomaterials Applications for Nanomedicine, R. Pignatello, Ed., chapter 17, pp. 333-358, InTech, Rijeka, Croatia, 2011.

[16] V. Trandafir, G. Popescu, M. G. Albu, H. Iovu, and M. Georgescu, Bioproducts Based on Collagen, Ars Docendi Publishing House, 2007.

[17] T. Kobayashi, S. Nakamura, and K. Yamashita, "Enhanced osteobonding by negative surface charges of electrically polarized hydroxyapatite," Journal of Biomedical Materials Research, vol. 57, no. 4, pp. 477-484, 2001.

[18] R. Injac, V. Djordjevic-Milic, and B. Srdjenovic, “Thermostability testing and degradation profiles of doxycycline in bulk, tablets, and capsules by HPLC," Journal of Chromatographic Science, vol. 45, no. 9, pp. 623-628, 2007.

[19] B. A. Cunha, C. M. Sibley, and A. M. Ristuccia, "Doxycycline," Therapeutic Drug Monitoring, vol. 4, no. 2, pp. 115-135, 1982.

[20] M. Walter, M. Frank, M. Satue et al., "Bioactive implant surface with electrochemically bound doxycycline promotes bone formation markers in vitro and in vivo," Dental Materials, vol. 30, no. 4, pp. 463-463, 2014.

[21] E. Kütan, G. Duygu-Çapar, C. Özçakir-Tomruk et al., "Efficacy of doxycycline release collagen membrane on surgically created and contaminated defects in rat tibiae: a histopathological and microbiological study," Archives of Oral Biology, vol. 63, pp. 1521, 2016.

[22] M. G. Albu, M. V. Ghica, M. Leca et al., "Doxycycline delivery from collagen matrices crosslinked with tannic acid," Molecular Crystals \& Liquid Crystals, vol. 523, pp. 97/[669]-105/[677], 2010.

[23] I. Titorencu, M. G. Albu, M. Giurginca et al., "In vitro biocompatibility of human endothelial cells with collagen-doxycycline matrices," Molecular Crystals and Liquid Crystals, vol. 523, no. 1, pp. 82/654-96/668, 2010.

[24] M. G. Albu, E. Kutan, M. Veshler, C. Ozcakir-Tomruk, I. Cristescu, and M. V. Ghica, "Efficacy of Doxicoll-a novel drug delivery systems for infected bone tissue," European Cell and Materials, vol. 30, supplement 2, p. 39, 2015.

[25] S. Marin, M. M. Marin, A.-M. Ene et al., "Collagen-doxycycline spongious forms for infected tissues treatment," in Proceedings of the 5th International Conference on Advanced Materials and Systems (ICAMS '14), pp. 249-254, Bucharest, Romania, October 2014. 

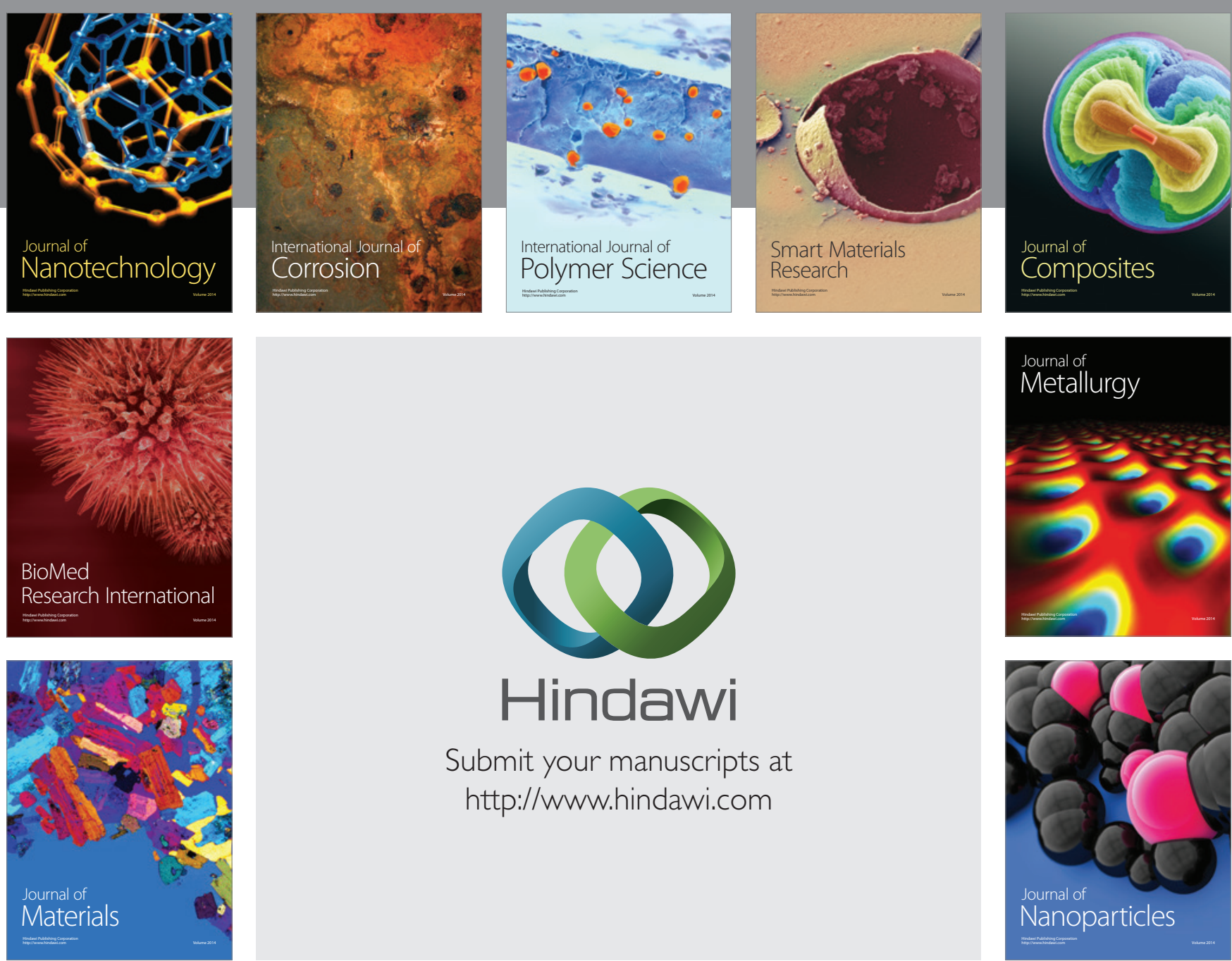

\section{Hindawi}

Submit your manuscripts at

http://www.hindawi.com

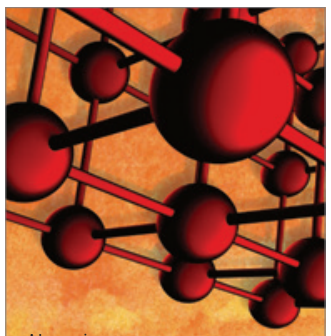

Materials Science and Engineering
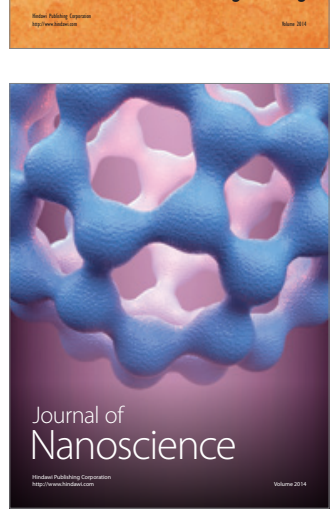
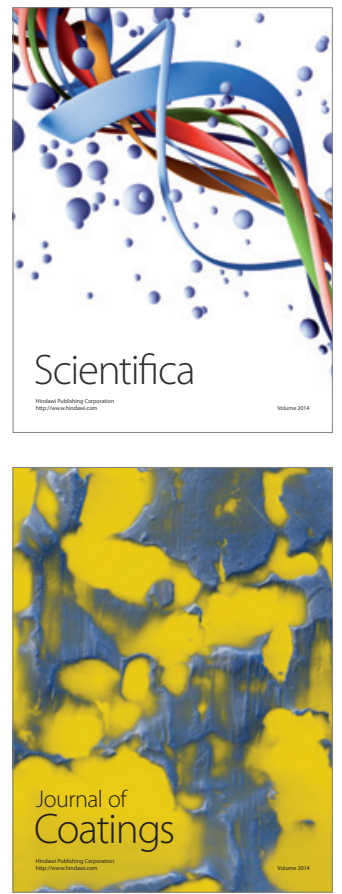
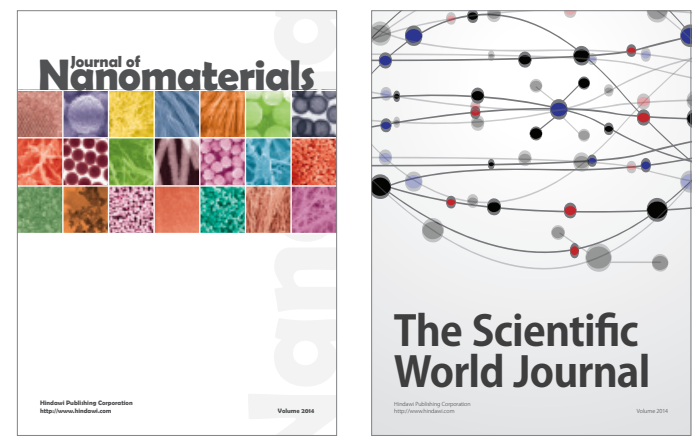

The Scientific World Journal
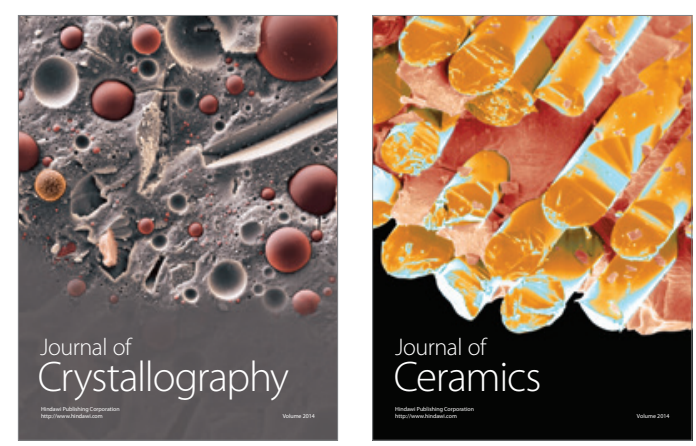
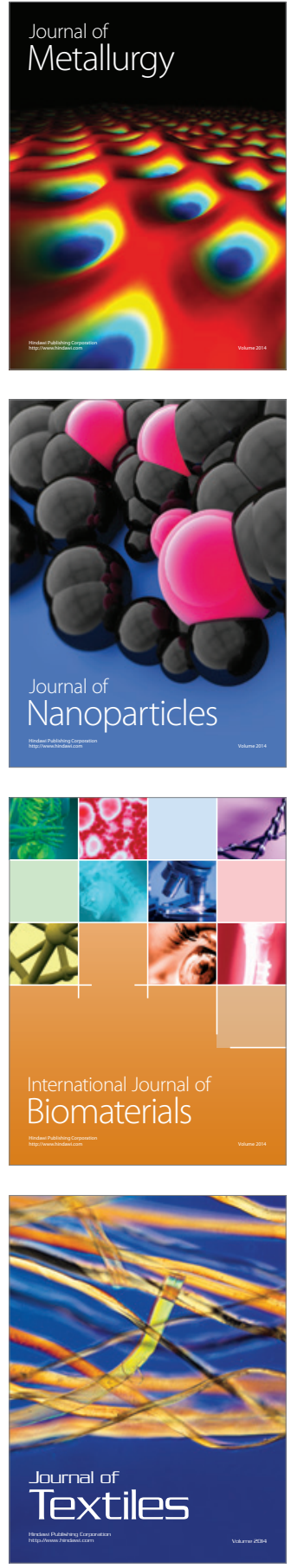